\title{
Risperidone-Induced Renal Damage and Metabolic Side Effects: The Protective Effect of Resveratrol
}

\author{
Sedat Bilgiç, ${ }^{1}$ Deniz Taștemir Korkmaz, ${ }^{1}$ Sebile Azirak, ${ }^{1}$ Ayșe Nilay Güivenç, \\ Nevin Kocaman, ${ }^{2}$ and Mehmet Kaya Özer ${ }^{3}$ \\ ${ }^{1}$ Vocational School of Health Services, Adiyaman University, Adiyaman, Turkey \\ ${ }^{2}$ Department of Histology, Faculty of Medicine, Firat University, Elazı̆̆, Turkey \\ ${ }^{3}$ Department of Pharmacology, Faculty of Medicine, Adryaman University, Adryaman, Turkey
}

Correspondence should be addressed to Sedat Bilgiç; sbilgic@adiyaman.edu.tr

Received 5 March 2017; Accepted 26 April 2017; Published 15 June 2017

Academic Editor: Ersin Fadillioglu

Copyright (c) 2017 Sedat Bilgiç et al. This is an open access article distributed under the Creative Commons Attribution License, which permits unrestricted use, distribution, and reproduction in any medium, provided the original work is properly cited.

\begin{abstract}
Objective. The aim of the study was to investigate the possible protective qualities of resveratrol (RSV) against the side effects of risperidone (RIS) in an experimental model in rat kidneys with histologic and biochemical assessments. Materials and Methods. Experimental procedures were performed on 35 female Sprague Dawley rats. Rats were randomly divided into five groups: control, untreated rats $(n=7)$ were in group 1; group 2 was given $2 \mathrm{mg} / \mathrm{kg} / \mathrm{day}$ RIS $(n=7)$; group 3 was treated with $2 \mathrm{mg} / \mathrm{kg} /$ day RIS and $20 \mathrm{mg} / \mathrm{kg} /$ day RSV ( $n=7)$; group 4 was treated with $2 \mathrm{mg} / \mathrm{kg} / \mathrm{day}$ RIS and $40 \mathrm{mg} / \mathrm{kg} / \mathrm{day}$ RSV $(n=7)$; and group 5 was treated with $2 \mathrm{mg} / \mathrm{kg} /$ day RIS and $80 \mathrm{mg} / \mathrm{kg} /$ day RSV $(n=7)$. All treatments were administered for two weeks by gavage. On treatment day 15, kidney tissues were removed for analysis. Results. The results showed that RSV treatment reduced weight gain induced by RIS. In addition, RSV increased the total antioxidant status (TAS) and decreased serum creatinine (Cr), blood urea nitrogen (BUN), oxidative stress index (OSI), and total oxidant status (TOS) levels significantly $(p<0.05)$. Conclusion. This study revealed that treatment with RSV might protect kidney tissues against the side effects of RIS. RSV could be an effective course of therapy to enhance therapeutic efficacy.
\end{abstract}

\section{Introduction}

RIS, structurally, a benzisoxazole derivative, is one of the most frequently prescribed atypical antipsychotics in the management of schizophrenia, mood disorders, and autism [1]. Its mechanism of action is not entirely clear. Although the use of atypical antipsychotic drugs has been successful in the treatment of schizophrenia, they can provoke some complications, including weight gain, sedation, movement problems, sleepiness, vision difficulties, constipation, hyperprolactinemia, and extrapyramidal side effects [2]. Patients using these drugs tend to disrupt treatment primarily due to their adverse effects. In addition, RIS is a highly potent drug, extensively metabolized in the liver and excreted by the kidneys [3]. RIS and its primary active metabolite, 9-hydroxyrisperidone, are eliminated by the kidneys. In patients with moderate to severe renal disease, clearance of the sum of the parent drug and metabolite has been demonstrated to decrease by $60 \%$ compared to healthy subjects [4].

There are a few cases reporting atypical antipsychotic drug-associated renal damage in patients (including those using RIS), but the mechanism that leads to RIS-related injury is not well understood [5]. Adverse outcomes potentially attributable to these drugs, such as hypotension, acute urinary retention, and rhabdomyolysis, are known to cause this injury [6]. Moreover, pneumonia, acute myocardial infarction, and ventricular arrhythmia have been associated with these drugs in former population-based studies and renal damage may also co-occur with these events [7].

Nowadays, medicinal plants are a considerable source of drug synthesis. RSV, trans-3,5,4'-trihydroxy stilbene, has potent antioxidative and anti-inflammatory properties [8]. It is a polyphenolic phytoalexin present in many edible plants, including mulberries, peanuts, and grapes [9]. RSV 
has been demonstrated to exhibit a wide range of healthpromoting benefits for the coronary, neurological, hepatic, and cardiovascular systems. It has been shown to inhibit inflammation, viral infection, oxidative stress, platelet aggregation, and the growth of a variety of cancer cells [10]. In addition, RSV has been studied in vivo and in vitro [11] and has been shown to possess a series of pharmacological benefits, including nephroprotective effects [12], as a result of its antioxidant and cytoprotective properties [13]. The antioxidant properties of RSV are mainly dependent upon the upregulation of endogenous cellular antioxidant systems, but the compound also displays direct reactive oxygen species (ROS) scavenging properties [14]. The high potency and low systemic toxicity of RSV make it a promising alternative to conventional therapeutic drugs. The fact that RIS exposure induces an excessive increase in metabolic alterations suggests that RSV could be used as an alternative therapy.

To our knowledge, there is no report regarding the protective and therapeutic effects of RSV on RIS-induced renal damage because it has not been studied until now. Therefore, the present study was designed to investigate the possible beneficial impact of oral supplementation with RSV against RIS-induced renal damage in rats for the first time. To achieve our goal, we performed several biochemical and histological analyses in female rats.

\section{Materials and Methods}

2.1. Chemicals. RIS was bought from Janssen Turkey, Istanbul, and RSV (CAS number 501-36-0) was purchased from Sigma-Aldrich (St. Louis, MO, USA). They were dissolved in distilled water. All other chemicals used were of the best analytical grade.

2.2. Animals. In this study, thirty-five female Sprague Dawley rats were used. Healthy adult (twelve weeks old) rats weighing 250-300 g were obtained from the Firat University Laboratory Animal Production and Research Center. All animal care and follow-up procedures were performed at this center. The experiments were performed in accordance with the protocol approved by Firat University Faculty of Medicine, Laboratory Animals Ethics Committee (protocol number $2016 / 25)$. The rats were kept at $21 \pm 1^{\circ} \mathrm{C}$ for $12 \mathrm{~h}$ in a lightdark cycle, were fed standard rat chow, and drank tap water ad libitum. RIS and RSV were administered to the rats for two weeks.

2.3. Experimental Design. In our study, 35 rats were randomly assigned to five groups with an equal number in each group. We used the simple randomization technique in this experimental study. Thirty-five female Sprague Dawley rats were divided into five groups as follows: group 1, control; group 2, RIS; group 3, RIS+RSV-1; group 4, RIS+RSV-2; and group 5, RIS+RSV-3. The control group was given physiological saline solution by gavage once a day. $2 \mathrm{mg} / \mathrm{kg} / \mathrm{day}$ RIS was administered by gavage for two weeks to all groups, except the control group. $20 \mathrm{mg} / \mathrm{kg} /$ day RSV was given for two weeks to the RIS+RSV-1 group. $40 \mathrm{mg} / \mathrm{kg} /$ day RSV was given for two weeks to the RIS+RSV-2 group. $80 \mathrm{mg} / \mathrm{kg} / \mathrm{day}$
RSV was given for two weeks to the RIS+RSV-3 group. All compounds were suspended in physiological saline solution and administered by gavage once a day. Body weight was recorded at the beginning and end of the study. The treatment course lasted two weeks for all groups. At the end of the second week of the treatment period, the animals were euthanized by exsanguination through cardiac puncture under diethyl ether anesthesia. Before killing, rats were individually weighed, venous blood samples were collected, and serum samples for biochemical analysis were separated by centrifuge at $2800 \mathrm{~g}$ for $15 \mathrm{~min}$ and then stored at $-80^{\circ} \mathrm{C}$. Kidneys were surgically removed, weighed, and stored at $-80^{\circ} \mathrm{C}$ for subsequent biochemical and pathohistological analysis.

The daily dose of RIS varies between 0.25 and $16 \mathrm{mg}$, and a frequently prescribed dose in previous studies was $2 \mathrm{mg}$ [15]. The maximum recommended human dose (MRHD) of RIS is $0.4-12 \mathrm{mg} /$ day. The experimental dose was calibrated as $2.0 \mathrm{mg} / \mathrm{kg}(\mathrm{MHRD} \times 10)$ on the basis of per $\mathrm{kg}$ body weight per day and its suitability to the rat animal model [16]. Therefore, a $2 \mathrm{mg}$ dose was selected for the present study. The assigned dosage of powdered RIS ( $2 \mathrm{mg} / \mathrm{kg}$ once a day for two weeks) was administered by a gastric tube daily between 8:00 and 9:00 a.m. in line with a previous report [17]. RSV $(20,40$, and $80 \mathrm{mg} / \mathrm{kg}$ body weight/day) was also administered by a gastric tube daily between 8:00 and 9:00 a.m. The dose and duration of RSV were selected according to results from a previous study [18].

2.4. Biochemical Analysis. BUN was measured using a commercially available enzymatic colorimetric method and analyzer system (Hitachi 917 modular device; Roche Diagnostics, Basel, Switzerland). Serum Cr was measured by Jaffe's method using the same analyzer system [19].

2.5. TAS and TOS Determination. The automated calorimetric measurement methods developed by Erel were used to define the TAS $(\mathrm{mmol} / \mathrm{L})$ and TOS $(\mu \mathrm{mol} / \mathrm{L}$ ) (a serum oxidant parameter). The measurement of TAS and TOS in serum samples was determined by a TAS and TOS kit (REL Assay Diagnostics) [20, 21].

2.6. OSI Determination. OSI was determined as TOS to TAS ratio and was calculated as follows: OSI (arbitrary unit) = ((TOS, $\mu \mathrm{mol} \mathrm{H} 2 \mathrm{O} 2 \mathrm{eq} / \mathrm{L}) /(\mathrm{TAS}, \mu \mathrm{mol}$ Trolox eq/L)) [22].

2.7. Terminal Deoxynucleotidyl Transferase dUTP Nick End Labeling (TUNEL) Assay. Apoptotic cells were defined using the ApopTag Plus Peroxidase In Situ Apoptosis Detection Kit (Chemicon, cat. number: S7101, USA) according to the manufacturer's instructions. Sections $(5 \mu \mathrm{m})$ taken from the paraffin blocks were entrenched onto polylysine-coated slides, deparaffinized using xylene, dehydrated with a series of alcohol rinses, and then washed with phosphate-buffered saline (PBS). Then, tissues were incubated with a proteinase $\mathrm{K}$ solution $(0.05 \%)$ and with $3 \%$ hydrogen peroxide for $5 \mathrm{~min}$ to forestall endogenous peroxidase activity. After washing with PBS, the tissues were incubated with Equilibration Buffer for $6 \mathrm{~min}$ and in working solution $(70 \% \mu \mathrm{L}$ reaction buffer $+30 \% \mathrm{TdT}$ enzyme) at $37^{\circ} \mathrm{C}$ under moist conditions for $60 \mathrm{~min}$. Tissues were then incubated in stop/wash 
TABLE 1: Body weight (g) of animals during treatment.

\begin{tabular}{|c|c|c|c|c|c|}
\hline Design of treatment & Control & RIS & RIS+RSV-1 & RIS+RSV-2 & RIS+RSV-3 \\
\hline Initial study & $238.28 \pm 7.53$ & $234.57 \pm 7.27$ & $225.28 \pm 4.16$ & $232.40 \pm 3.55$ & $244.80 \pm 9.88$ \\
\hline Final study & $252.85 \pm 7.46$ & $248.00 \pm 5.86$ & $233.71 \pm 3.68$ & $226.80 \pm 5.25$ & $246.80 \pm 9.42$ \\
\hline \multicolumn{3}{|c|}{ Statistical comparison (initial study versus final study) } & $(p)$ & & \\
\hline \multicolumn{3}{|c|}{ Control } & 0.000 & & \\
\hline \multicolumn{3}{|l|}{ RIS } & 0.005 & & \\
\hline \multicolumn{3}{|l|}{ RIS+RSV-1 } & 0.005 & & \\
\hline \multicolumn{3}{|l|}{ RIS+RSV-2 } & 0.071 & & \\
\hline \multicolumn{3}{|l|}{ RIS+RSV-3 } & 0.537 & & \\
\hline
\end{tabular}

Changes in the body weight of experimental rats. Values are expressed as mean \pm SEM of seven animals. The groups were compared with the paired-samples $t$ test at initial and final treatment. $p \leq 0.05$. RIS: risperidone; RSV: resveratrol; RIS+RSV-1: $2 \mathrm{mg} / \mathrm{kg}$ RIS $+20 \mathrm{mg} / \mathrm{kg} \mathrm{RSV} ; \mathrm{RIS}+\mathrm{RSV}-2: 2 \mathrm{mg} / \mathrm{kg} \mathrm{RIS}+40 \mathrm{mg} / \mathrm{kg}$ RSV; RIS+RSV-3: 2 mg/kg RIS+80 mg/kg RSV.

buffer for $10 \mathrm{~min}$ and incubated in anti-digoxigeninperoxidase for $30 \mathrm{~min}$. Apoptotic cells were examined using the diaminobenzidine substrate. Cross sections contrast stained with methyl green were sealed using a proper covering solution. Mamma tissue was used as a positive control. PBS was used instead of the TdT enzyme in the negative control tissue. Preparations were examined and assessed using a research microscope (Olympus $\mathrm{BH} 2$ light microscope) and then photographed. To assess the TUNEL staining, after staining with methyl, green cells with green nuclei were considered normal whereas cells with brown nuclei were considered apoptotic. Apoptotic (TUNEL positive) cells were counted and statistically assessed. This analysis was made in at least eight areas of each kidney section (two sections/animal), and the sections were analyzed at $400 \mathrm{x}$ magnification [23].

The evaluation of TUNEL staining was made based on the extent of the staining of apoptotic cells. The extent of TUNEL staining was scored semiquantitatively as 0 (none), 1 (light), 2 (medium), and 3 (intense) [24].

2.8. Statistical Analyses. Statistical analysis was performed using SPSS 16.0 (SPSS, Chicago, IL, USA). All data were expressed as mean values \pm their standard errors (SEM). Normality for variables in the groups was determined by the Shapiro-Wilk test. For the comparison of the mean weight of all groups, a paired $t$-test was performed. The groups were compared with the paired-samples $t$-test at the beginning and end of the treatment. One-way analysis of variance (ANOVA) followed by the LSD post hoc test were used for the comparison of biochemical parameters and total oxidant/antioxidant levels. Significance was considered at the $p<0.05$ level. For histopathological analysis, results were expressed as the means \pm standard deviation (SD). The statistically significant difference was determined by ANOVA followed by Tukey's multiple comparison test. Probability values $(p)$ less than 0.05 were considered to be statistically significant.

\section{Results}

3.1. Effects of RIS and RSV on Weight Gain/Loss. Body weight measurements showed that during the two weeks, animals grew from $238.28 \mathrm{~g}$ at day one to $252.85 \mathrm{~g}$ for the control group, from $234.57 \mathrm{~g}$ at day one to $248.00 \mathrm{~g}$ for the RIS group, from $225.28 \mathrm{~g}$ at day one to $233.71 \mathrm{~g}$ for the RIS+RSV-1 group, from $232.40 \mathrm{~g}$ at day one to $226.80 \mathrm{~g}$ for the RIS +RSV-2 group, and from $244.80 \mathrm{~g}$ at day one to $246.80 \mathrm{~g}$ for the RIS+RSV-3 group at day 14 (Table 1: paired-samples $t$-test for the body weight at day $14 ; p=0.000, p=0.005$, $p=0.005, p=0.071$, and $p=0.537$, resp.).

There was a significantly increased total body weight gain in the control, RIS, and RIS+RSV-1 treatment groups $(p=0.000, p=0.005$, and $p=0.005$, resp.). However, the RIS+RSV-2 group was observed to have a decreased weight gain and the RIS+RSV-3 group an increased weight gain had no significant effect on these measurements $(p=0.071$, $p=0.537$, resp.) (Table 1 ; Figure 1 ).

3.2. Effects of RIS and RSV on Biochemical and Oxidative Stress Parameters. We measured levels of biochemical parameters in serum, the results are shown in Table 2, and oxidative stress parameters are shown in Table 3. Serum Cr level was significantly increased in the RIS group compared to the control, RIS+RSV-1, RIS+RSV-2, and RIS+RSV-3 groups $(p<0.00)$. Serum $\mathrm{Cr}$ level was significantly increased in the RIS+RSV-1 group compared to the RIS+RSV-2 and RIS+RSV-3 groups $(p=0.01, p=0.00$, resp.) (Table 2; Figure 2).

BUN level was significantly increased in the RIS group compared to the control, RIS+RSV-1, and RIS+RSV-2 groups $(p=0.000, p=0.000$, and $p=0.001$, resp.). BUN level was significantly decreased in the RIS+RSV-1 group compared to the RIS, RIS+RSV-2, and RIS + RSV -3 groups $(p=0.000, p=0.000$, and $p=0.000$, resp.) (Table 2; Figure 2).

Ameliorative effects of RSV treatment against RIS administration significantly increased the TAS level and decreased TOS and OSI levels $(p<0.05)$. The control group had a significantly higher TAS level compared to the RIS group $(p=0.014)$. The RIS+RSV-1 group had a significantly higher TAS level compared to the control, RIS, RIS+RSV-2, and RIS+RSV-3 groups $(p=0.037, p=0.000, p=0.002$, and $p=0.000$, resp.). The RIS $+\mathrm{RSV}-2$ group had a significantly higher TAS level compared to the RIS+RSV-3 groups $(p=0.011)$. TOS level was significantly higher in the RIS 


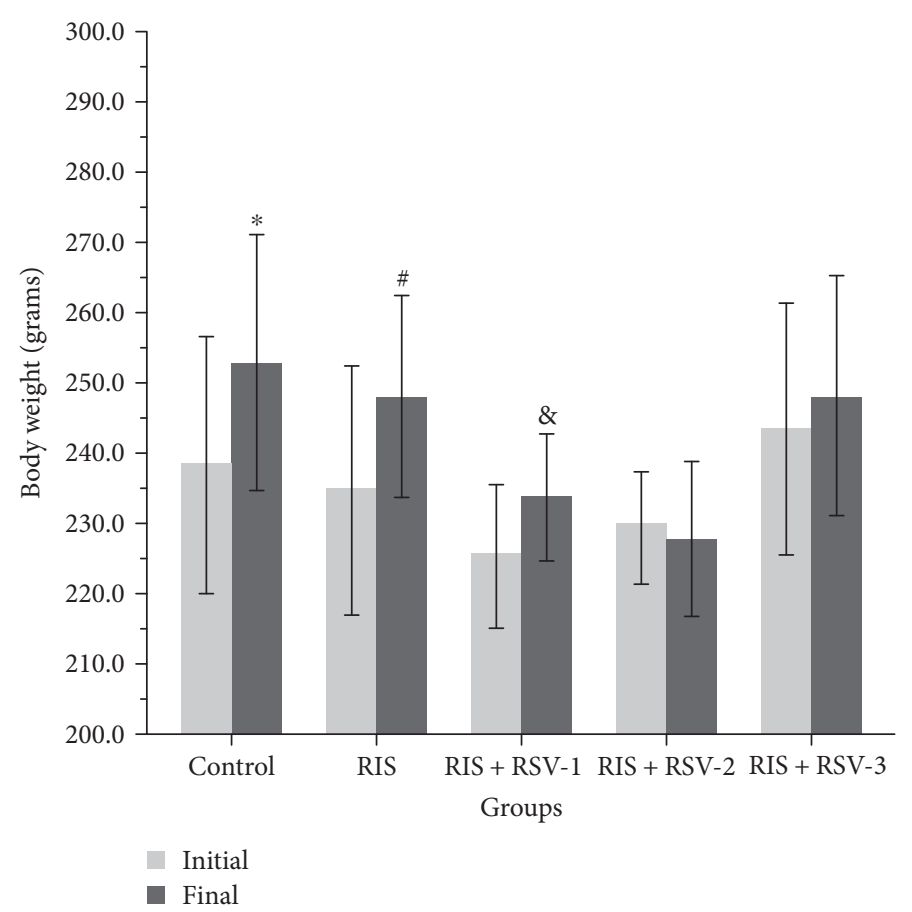

FIGURE 1: Changes in the body weight of experimental rats. Values are expressed as mean \pm SEM of seven animals. The groups were compared with the paired-samples $t$-test at initial and final treatment. ${ }^{*, \#, ~ \& ~ I n ~ e a c h ~ c o l u m n, ~ d i f f e r e n t ~ s u p e r s c r i p t ~ c h a r a c t e r s ~ m e a n ~ s i g n i f i c a n t ~ d i f f e r e n c e s ~}$ at $p<0.05$ in different groups. Abbreviations: RIS, risperidone; RSV, resveratrol; RIS+RSV-1, 2 mg/kg RIS+20 mg/kg RSV; RIS+RSV-2, 2 mg/kg RIS+40 mg/kg RSV; RIS+RSV-3, 2 mg/kg RIS+80 mg/kg RSV.

TABLE 2: Levels of serum biochemical parameters for all groups.

\begin{tabular}{|c|c|c|c|c|c|c|}
\hline Parameters & Control & RIS & RIS+RSV-1 & RIS+RSV-2 & RIS+RSV-3 & $p$ \\
\hline Scr (mg/dL) & $0.46 \pm 0.00^{\mathrm{b}, \mathrm{d}, \mathrm{e}}$ & $0.53 \pm 0.00^{\mathrm{a}, \mathrm{c}, \mathrm{d}, \mathrm{e}}$ & $0.45 \pm 0.01^{\mathrm{b}, \mathrm{d}, \mathrm{e}}$ & $0.38 \pm 0.01^{\mathrm{a}, \mathrm{b}, \mathrm{c}}$ & $0.37 \pm 0.01^{\mathrm{a}, \mathrm{b}, \mathrm{c}}$ & 0.000 \\
\hline BUN (mg/dL) & $18.71 \pm 0.68^{\mathrm{b}, \mathrm{d}, \mathrm{e}}$ & $25.85 \pm 0.45^{\mathrm{a}, \mathrm{c}, \mathrm{d}}$ & $19.85 \pm 0.50^{\mathrm{b}, \mathrm{d}, \mathrm{e}}$ & $23.28 \pm 0.42^{\mathrm{a}, \mathrm{b}, \mathrm{c}}$ & $24.57 \pm 0.48^{\mathrm{a}, \mathrm{c}}$ & 0.000 \\
\hline
\end{tabular}

Each group represents the mean \pm SEM for seven rats. ${ }^{a} p<0.01$ versus the control group; ${ }^{b} p<0.01$ versus the RIS group; ${ }^{c} p<0.01$ versus the RIS+RSV-1 group; $\mathrm{d}_{p}<0.01$ versus the RIS+RSV-2 group; and ${ }^{\mathrm{e}} p<0.01$ versus the RIS+RSV-3 group. RIS: risperidone; RSV: resveratrol; Scr: serum creatinine; BUN: blood urea nitrogen; RIS+RSV-1: 2 mg/kg RIS+20 mg/kg RSV; RIS+RSV-2: 2 mg/kg RIS+40 mg/kg RSV; RIS+RSV-3: 2 mg/kg RIS+80 mg/kg RSV.

TABLE 3: Comparison of serum oxidative stress parameters among the groups.

\begin{tabular}{|c|c|c|c|c|c|c|}
\hline Parameters & Control & RIS & RIS+RSV-1 & RIS+RSV-2 & RIS+RSV-3 & $p$ \\
\hline TOS $(\mu \mathrm{mol} / \mathrm{L})$ & $9.74 \pm 0.71^{\mathrm{b}, \mathrm{e}}$ & $16.04 \pm 1.03^{\mathrm{a}, \mathrm{c}, \mathrm{d}, \mathrm{e}}$ & $10.45 \pm 1.06^{\mathrm{b}, \mathrm{e}}$ & $8.19 \pm 0.56^{\mathrm{b}}$ & $6.27 \pm 1.09^{\mathrm{a}, \mathrm{b}, \mathrm{c}}$ & 0.000 \\
\hline TAS $(\mathrm{mmol} / \mathrm{L})$ & $1.60 \pm 0.39^{\mathrm{b}, \mathrm{c}, \mathrm{e}}$ & $0.78 \pm 0.14^{\mathrm{a}, \mathrm{c}}$ & $2.28 \pm 0.23^{\mathrm{a}, \mathrm{b}, \mathrm{d}, \mathrm{e}}$ & $1.23 \pm 0.10^{c, e}$ & $0.38 \pm 0.03^{\mathrm{a}, \mathrm{c}, \mathrm{d}}$ & 0.000 \\
\hline OSI (AU) & $791.59 \pm 162.39^{b}$ & $2805.78 \pm 857.32^{\mathrm{a}, \mathrm{c}, \mathrm{d}}$ & $464.05 \pm 25.47^{\mathrm{b}, \mathrm{e}}$ & $692.31 \pm 69.32^{\mathrm{b}}$ & $1797.28 \pm 452.35^{\mathrm{c}}$ & 0.004 \\
\hline
\end{tabular}

Each group represents the mean \pm SEM for seven rats. ${ }^{a} p<0.04$ versus the control group; ${ }^{b} p<0.02$ versus the RIS group; ${ }^{c} p<0.05$ versus the RIS+RSV-1 group; ${ }^{\mathrm{d}} p<0.03$ versus the RIS+RSV-2 group; and ${ }^{\mathrm{e}} p<0.05$ versus the RIS+RSV-3 group. RIS: risperidone; RSV: resveratrol; TAS: total antioxidant status; TOS: total oxidant status; OSI: oxidative stress index; RIS+RSV-1: 2 mg/kg RIS+20 mg/kg RSV; RIS+RSV-2: 2 mg/kg RIS+40 mg/kg RSV; RIS+RSV-3: 2 mg/kg RIS+80 mg/kg RSV; AU: arbitrary units.

group compared to the control, RIS+RSV-1, RIS+RSV-2, and RIS+RSV-3 groups $(p<0.001)$. The RIS+RSV-1 group had a significantly higher TOS level compared to the RIS+RSV-3 group $(p=0.003)$. OSI level was significantly higher in the RIS group compared to the control, RIS+RSV-1, and RIS+ RSV-2 groups $(p=0.003, p=0.001$, and $p=0.002$, resp.) (Table 3; Figure 3).
3.3. Evaluation of Apoptosis in Kidney Tissues. The results of the apoptotic index are shown in Table 4 and Figure 4. Using TUNEL assay to detect apoptotic renal tubular cells in the kidney sections, the control group (Figure 4(a)) showed only a few TUNEL-positive cells. The number of TUNEL-positive cells markedly increased in the RIS group (Figure 4(b)) compared with the control group $(p<0.05)$. RIS + RSV -1 

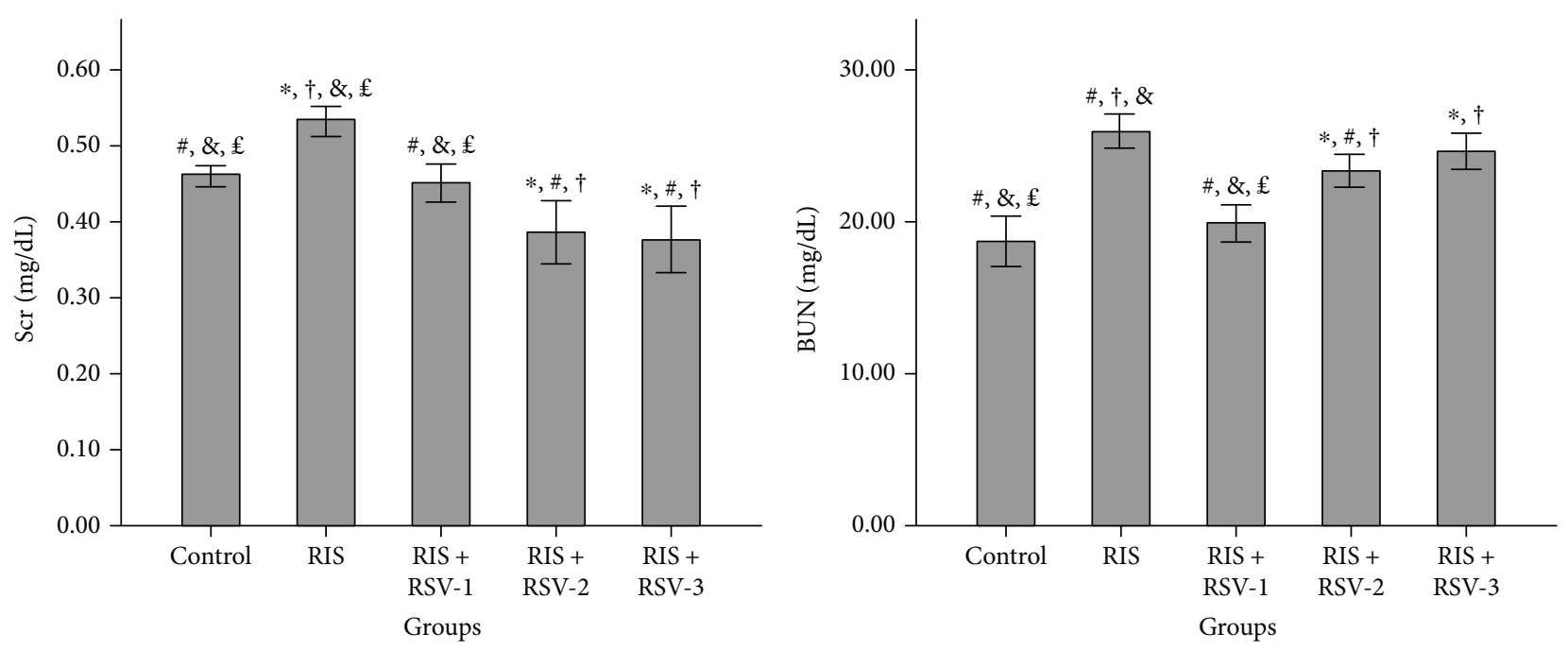

FIGURE 2: Effects of risperidone, resveratrol, and their coadministration on the kidney level of serum creatinine and blood urea nitrogen in rats after two weeks. Values are expressed as mean \pm SEM of seven animals. ANOVA followed by the LSD post hoc test were used. ${ }^{*} p<0.05$ versus control; ${ }^{\#} p<0.05$ versus RIS-treated rats; ${ }^{\dagger} p<0.05$ versus RIS+RSV-1-treated rats; ${ }^{\&} p<0.05$ versus RIS+RSV-2-treated rats; and ${ }^{\circledR} p<0.05$ versus RIS+RSV-3-treated rats. Abbreviations: RIS, risperidone; RSV, resveratrol; Scr, serum creatinine; BUN, blood urea nitrogen; RIS +RSV-1, 2 mg/kg RIS+20 mg/kg RSV; RIS+RSV-2, 2 mg/kg RIS+40 mg/kg RSV; RIS+RSV-3, 2 mg/kg RIS+80 mg/kg RSV.

(Figure 4(c)), RIS+RSV-2 (Figure 4(d)), and RIS+RSV-3 (Figure $4(\mathrm{e})$ ) groups were similar and showed rare TUNELpositive cells. Treatment with RSV (RIS+RSV-1, RIS+RSV2, and RIS+RSV-3 groups) (Figures 4(c), 4(d), and 4(e)) reduced the number of TUNEL-positive cells as compared with the RIS group $(p<0.05)$.

\section{Discussion}

Certain herbal medicines have been declared to be effective in the treatment of adverse outcomes attributed to atypical antipsychotic drugs, including RIS, and combination treatment with drugs and herbal medicines has been reported to be useful in enhancing treatment influence and reducing recovery time and adverse effects. In the present study, RIS $(2 \mathrm{mg} /$ $\mathrm{kg} /$ day) plus RSV (20, 40 and $80 \mathrm{mg} / \mathrm{kg} /$ day) was given by gavage to rats for two weeks. All doses of RSV caused dosedependent decreases in weight gain and alleviated renal damage of the animals compared to the group that received only RIS. Thus, the present study aimed to investigate whether RSV implemented a protective effect on RIS-induced renal damage and metabolic side effects in an experimental animal model.

Second-generation antipsychotics (SGAs) are extensively used in several psychiatric disease entities and exert to various extents the risk of antipsychotic-induced weight gain. In a study by Domecq et al., weight gain was associated with the use of RIS [25]. A meta-analysis by Allison et al. in adults estimated that the mean weight gain after 10 weeks of treatment was $2.00 \mathrm{~kg}$ for RIS [26]. Weight gain can impair both physical health and psychological well-being. Therefore, it is important to define factors that are associated with the risk of weight gain with atypical antipsychotics. These factors may include lifestyle issues, particularly diet. RSV, being a dietary constituent, is an excellent therapy candidate for disorders with a metabolic origin. Since several studies using animal models of diet-induced obesity have displayed the beneficial effects of RSV on reducing obesity, many clinical trials have been effected to assess its effect in humans. In a study by Gómez-Zorita et al., RSV decreased whole body weight in obese rats by reducing oxidative stress [27]. Dal-Pan et al. postulated resveratrol's facilitation of weight loss in nonhuman primate models of obesity by increasing the metabolic rate and suppressing torpor expression. In fact, RSV administration in primate models led to a $13 \%$ reduction in energy intake while increasing the resting metabolic rate by $29 \%$ [28]. In the current study, RSV cotreatment diminished antipsychotic-induced weight gain with a more obvious effect and significantly decreased only with a $20 \mathrm{mg} / \mathrm{kg}$ dose. These results imply that the mechanism of action for RSV occurs by increasing energy expenditure, directly reducing energy storage and inhibition of energy intake. This weight decrement effect of RSV is estimated to be in part attributable to its effects on adipocytes [29, 30]. Hence, RSV, a dietary supplement, significantly prevents RIS-induced weight gain, which might suggest a potential effectiveness on human subjects. Therefore, RSV is a safe compound for coadministration with RIS for mitigating antipsychotic-induced weight gain/obesity without influencing their therapeutic action. In addition, these results are certain to influence the choice of protective compound when several options exist and to institute preemptive strategies for weight management.

Several adverse outcomes attributed to atypical antipsychotic drugs are known to cause renal damage. RIS was also described as increasing the risk of this damage in a population study [31]. Serum Cr and BUN were the classical standards to evaluate renal damage [32]. Accordingly, a study conducted by Hsu et al. demonstrated that elevated serum $\mathrm{Cr}$ was observed in RIS administration [33]. Therefore, the 

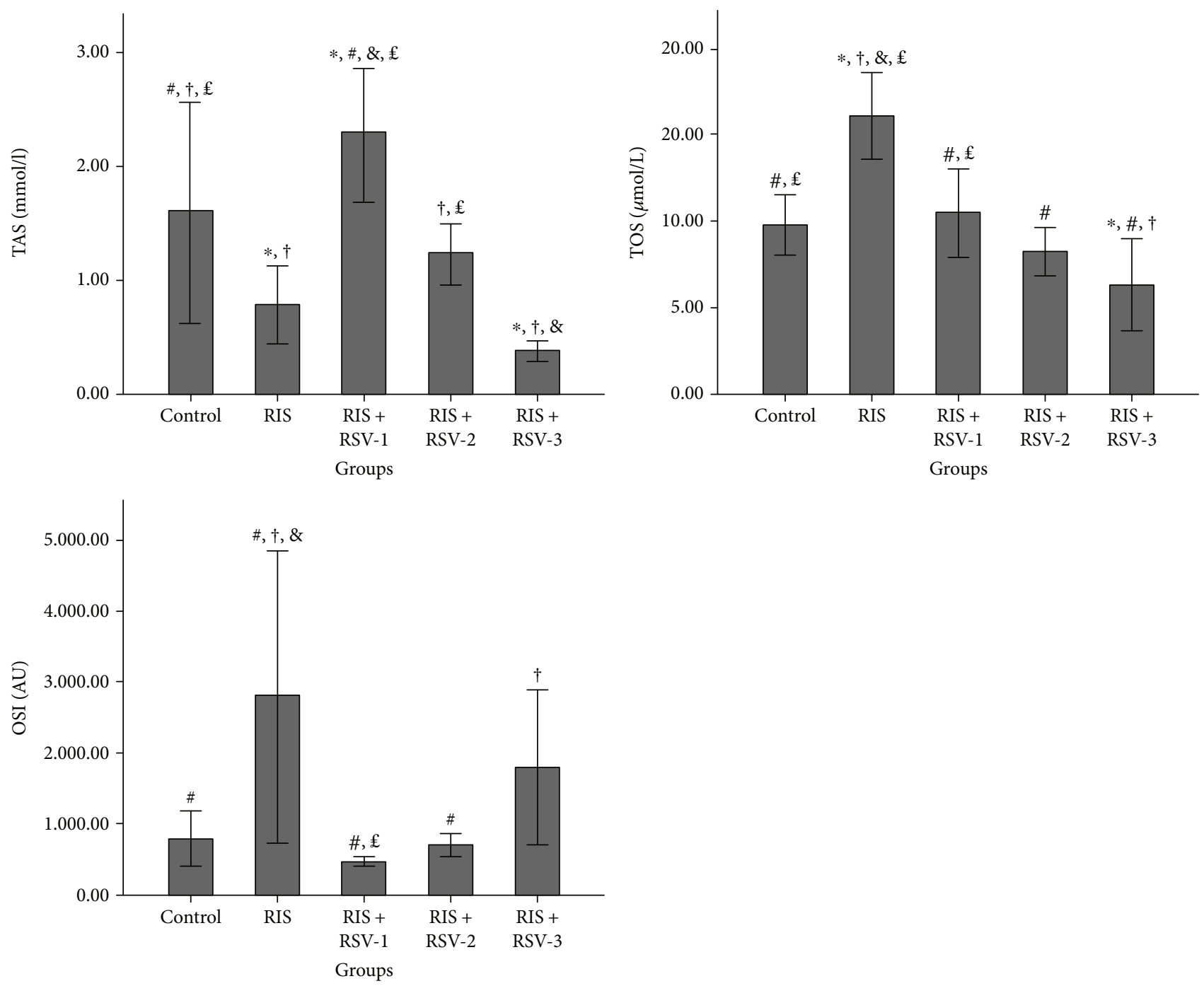

FIGURE 3: Effects of risperidone, resveratrol, and their coadministration on the level of TAS, TOS, and OSI in rats after two weeks. Values are expressed as mean \pm SEM of seven animals. ANOVA followed by the LSD post hoc test were used. ${ }^{*} p<0.05$ versus control; ${ }^{*} p<0.05$ versus RIS-treated rats; ${ }^{\dagger} p<0.05$ versus RIS+RSV-1-treated rats; ${ }^{\circledR} p<0.05$ versus RIS+RSV-2-treated rats; and ${ }^{£} p<0.05$ versus RIS+RSV-3-treated rats. Abbreviations: RIS, risperidone; RSV, resveratrol; TAS, total antioxidant status; TOS, total oxidant status; OSI, oxidative stress index; RIS +RSV-1, 2 mg/kg RIS+20 mg/kg RSV; RIS+RSV-2, 2 mg/kg RIS+40 mg/kg RSV; RIS+RSV-3, 2 mg/kg RIS+80 mg/kg RSV; AU: arbitrary units .

TABLE 4: Effects of risperidone and resveratrol on apoptotic index (\%) in rat kidneys.

\begin{tabular}{lc}
\hline Groups & Apoptotic index $(\%)(\mathrm{AI} ;$ mean \pm SD) \\
\hline Control & $4.83 \pm 1.17^{\mathrm{b}, \mathrm{c}, \mathrm{d}, \mathrm{e}}$ \\
RIS & $32.50 \pm 3.56^{\mathrm{a}, \mathrm{c}, \mathrm{d}, \mathrm{e}}$ \\
RIS+RSV-1 & $14.16 \pm 2.40^{\mathrm{a}, \mathrm{b}}$ \\
RIS+RSV-2 & $14.20 \pm 2.86^{\mathrm{a}, \mathrm{b}}$ \\
RIS+RSV-3 & $12.00 \pm 2.60^{\mathrm{a}, \mathrm{b}}$ \\
\hline
\end{tabular}

The apoptotic index of all the groups. Values are mean \pm SD for seven rats in each group. ${ }^{\mathrm{a}}$ Significant from control; ${ }^{\mathrm{b}}$ Significant from RIS; ${ }^{\mathrm{c}}$ Significant from RIS+RSV-1; ${ }^{\mathrm{d}}$ Significant from RIS+RSV-2; and ${ }^{\mathrm{e}}$ Significant from RIS + RSV-3 ( $p \leq 0.05)$. RIS: risperidone; RSV: resveratrol; RIS+RSV-1: $2 \mathrm{mg} / \mathrm{kg}$ RIS+20 mg/kg RSV; RIS+RSV-2: 2 mg/kg RIS+40 mg/kg RSV; RIS+RSV-3: $2 \mathrm{mg} / \mathrm{kg}$ RIS $+80 \mathrm{mg} / \mathrm{kg}$ RSV. The extent of TUNEL staining was scored semiquantitatively as 0 (none), 1 (light), 2 (medium), and 3 (intense). study of $\mathrm{Hsu}$ et al. is an indicator to prove renal damage through the use of RIS. RIS may impair tubular function and result in several renal complications. As a polyphenolic phytoalexin, RSV has been reported to be useful in the prevention of numerous types of kidney disease [34, 35], druginduced renal damage [12], and ischemia-reperfusion and sepsis-induced kidney injuries [36]. Similarly, Wu et al. demonstrated that RSV effectively attenuated renal oxidative stress in the diabetic rat kidney [37]. A number of independent studies further explored the molecular mechanisms of RSV-mediated nephroprotection. The results of the present study demonstrate that RIS exposure produced a significant increase in the level of serum Cr and BUN indicating damaged structural and functional kidney integrity. Consistent with these observations, the beneficial effects of RSV were identified in the prevention of renal tubular damage and dysfunction. In addition, oral supplementation of RSV 


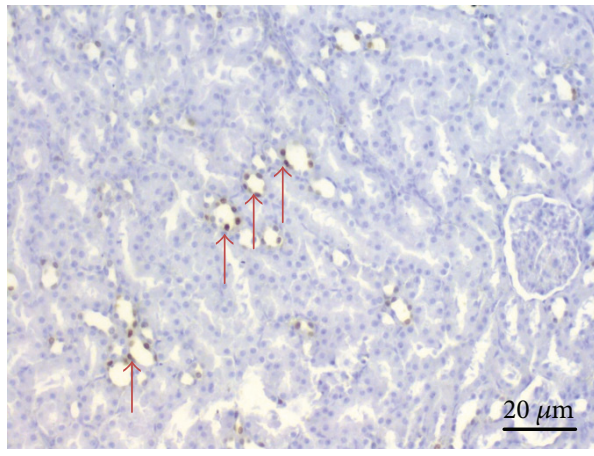

(a)

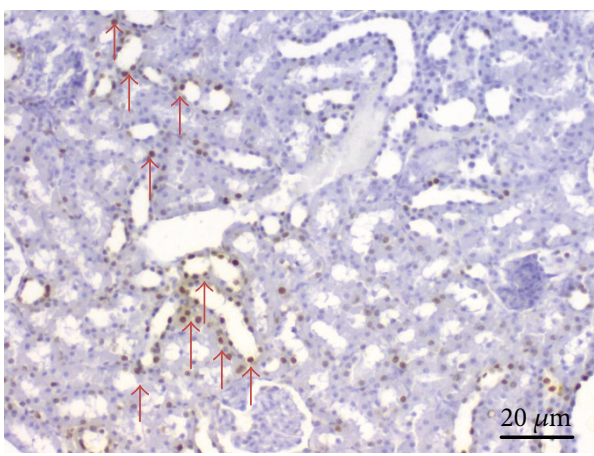

(c)

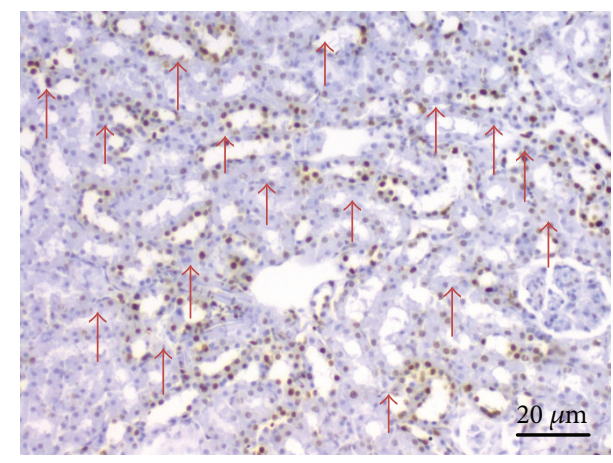

(b)

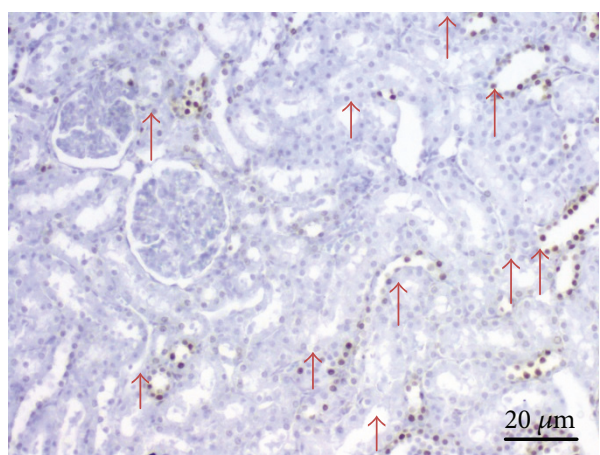

(d)

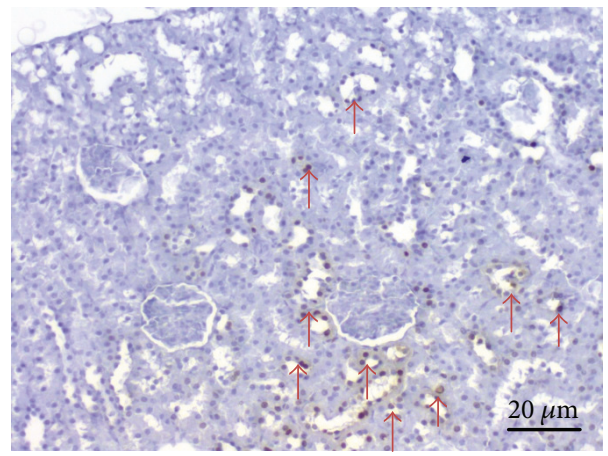

(e)

FIGURE 4: Representative photomicrographs of TUNEL staining in all five groups (scale bars $=20 \mu \mathrm{m}$ ). (a) Group 1 (control) only few TUNEL-positive cells (arrow). (b) Group 2 (RIS) a lot of TUNEL-positive cells (arrows). (c) Group 3 (RIS+RSV-1), (d) group 4 (RIS + RSV-2), and (e) group 5 (RIS+RSV-3) similarly rare TUNEL-positive cells (arrows). This analysis was exerted in at least eight areas of each kidney section (two sections/animal), and the sections were analyzed at 400x magnification. The evaluation of TUNEL staining was exerted based on the extent of the staining of apoptotic cells. The extent of TUNEL staining was scored semiquantitatively as 0 (none), 1 (light), 2 (medium), and 3 (intense).

demonstrated the restoration of the elevated serum $\mathrm{Cr}$ and BUN to the normal levels $[38,39]$. RSV cotreatment ameliorated these changes in all doses, with an especially obvious effect in high doses. These results are supported by data in the literature [40].

Oxidative stress is one of the key mechanisms responsible for renal damage and disease progression. Antioxidants try to fight oxidative stress and minimize its damage. TAS measurement has been used to evaluate the overall performance of the antioxidant system. TOS measurement provides a sensitive index of lipid peroxidation and oxidative stress [41]. The TOS/TAS ratio is termed "OSI," which is an indicator of the oxidative stress degree [42]. In the present study, RIS administration resulted in a decrease in TAS level and an increase in TOS and OSI levels as in former studies [43]. Conversely, we observed that RSV could protect kidneys from RIS-induced renal damage and provide useful changes in the basal levels of stress biomarkers. We found that the serum TAS level increased and the TOS and OSI levels prominently decreased with RSV treatment as reported in previous studies [44]. Similarly, Wong et al. demonstrated that RSV reduced oxidative damage biomarkers during aging in F2 hybrid mice [45]. In addition, RSV cotreatment ameliorated these changes with a more obvious effect in $20 \mathrm{mg} / \mathrm{kg}$ doses. The beneficial effects of RSV, observed in the current work, are very likely due to 
its strong antioxidant properties and may be associated with its constituent compounds. This implies that the RSV presented a nephroprotective activity probably due to its antioxidant capacity. The restoration of tissue antioxidant function by RSV may be attributed to its ability to upregulate antioxidant gene expression. Also, RSV may display its antinephrolithic properties by both inhibiting free radical formation and attenuating the expression of a number of inflammatory mediators [46].

Histological observations added more evidence of the protective effect of RSV. Apoptosis involves interactions among several protein families that regulate activation of various apoptotic markers. More recently, Elmorsy et al. demonstrated that some antipsychotics, including RIS, can induce apoptosis [47]. Apoptosis effects triggered by RIS appear to be cell-type dependent [48]. ROS can also induce cell death via apoptosis in many cell types. Such an effect was also blocked by RSV [49]. RSV is accepted as a potent antioxidant and antiapoptotic agent $[50,51]$. In the present study, histopathological evaluation of the kidneys showed severe damage ensued through loss of normal architecture, which included vacuolar degeneration and fatty changes in RISadministered rats. These toxic effects were effectively prevented by RSV treatment. Furthermore, RSV cotreatment ameliorated these changes with a more obvious effect in $80 \mathrm{mg} / \mathrm{kg}$ doses. The antiapoptotic effect of RSV based on the evaluated reduction in TUNEL-positive kidney cells may also contribute to its therapeutic impact against RIS. Therefore, the antioxidant activity of this compound may be a potential mechanism for antiapoptotic activity. Accordingly, RSV treatment of the cells against RIS exposure prevented apoptotic cell injury and death. The underlying mechanism of protection of RSV may be associated with the suppression of apoptosis via death receptor-mediated pathways. On the other hand, because the kidneys contain more mitochondria than the other organs, the nephrotoxic effects might also be due to the direct action of RIS on renal mitochondria. Therefore, the reducing effects of RIS on mitochondrial functions may also be important in the pathogenesis of nephrotoxicity $[52,53]$. Hence, it can be assumed that the antioxidant activity of RSV may be due to the effect on the mitochondria-dependent apoptotic pathway, but this has to be supported with further experimentation. Therefore, RSV may be a good option against RIS-induced side effects. The mechanism of action of RIS has not been fully explained, and its exact renal damage mechanism is still being researched because of insufficient experimental study in the literature histopathologically investigating the effects of RIS on renal cells in rats.

RSV was revealed to facilitate a significant reduction in serum $\mathrm{Cr}$ and BUN levels and to alleviate renal damage and dysfunction, which was further verified by apoptosis analysis of renal histology. In addition, RSV reduced weight gain, contributed to the oxygen radical scavenging activity, and increased antioxidant activity to accomplish its protective ability against damage caused by the side effects of RIS. Therefore, RSV may act through a range of mechanisms whose effects might have major therapeutic potential in RIS-induced side effects.
In conclusion, our results clearly indicate that RSV oral supplementation, in certain doses, protects against RISinduced renal damage. Therefore, RSV may be a clinically promising agent in RIS-induced renal damage and its metabolic side effects. Further studies should be undertaken to examine the potential effect of RSV on renal damage in human and animal models.

\section{Abbreviations}

BUN: Blood urea nitrogen

MRHD: Maximum recommended human dose

OSI: $\quad$ Oxidative stress index

PBS: $\quad$ Phosphate-buffered saline

RIS: $\quad$ Risperidone

ROS: Reactive oxygen species

RSV: Resveratrol

Cr: $\quad$ Creatinine

SGAs: Second-generation antipsychotic drugs

TAS: Total antioxidant status

TOS: Total oxidant status

TUNEL: Terminal deoxynucleotidyl transferase dUTP nick end labeling.

\section{Conflicts of Interest}

The authors have no conflict of interest.

\section{References}

[1] M. Dinnissen, A. Dietrich, B. J. Van Den Hoofdakker, and P. J. Hoekstra, "Clinical and pharmacokinetic evaluation of risperidone for the management of autism spectrum disorder," Expert Opinion on Drug Metabolism \& Toxicology, vol. 11, pp. 111-124, 2015.

[2] K. A. Stigler, M. N. Potenza, and C. J. McDougle, "Tolerability profile of atypical antipsychotics in children and adolescents," Pediatric Drugs, vol. 3, no. 12, pp. 927-942, 2001.

[3] W. Meuldermans, J. Hendrickx, G. Mannens et al., "The metabolism and excretion of risperidone after oral administration in rats and dogs," Drug Metabolism and Disposition, vol. 22, pp. 129-138, 1994.

[4] J. Heykants, M. L. Huang, G. Mannens et al., "The pharmacokinetics of risperidone in humans-a summary," The Journal of Clinical Psychiatry, vol. 55, pp. 13-17, 1994.

[5] V. Raitasuo, R. Vataja, and E. Elomaa, "Risperidone-induced neuroleptic malignant syndrome in young patient," Lancet, vol. 344, p. 1705, 1994.

[6] C. W. Ritchie, E. Chiu, S. Harrigan et al., "A comparison of the efficacy and safety of olanzapine and risperidone in the treatment of elderly patients with schizophrenia: an open study of six months duration," International Journal of Geriatric Psychiatry, vol. 21, pp. 171-179, 2006.

[7] W. A. Ray, C. P. Chung, K. T. Murray, K. Hall, and C. M. Stein, "Atypical antipsychotic drugs and the risk of sudden cardiac death," The New England Journal of Medicine, vol. 360, pp. 225-235, 2009.

[8] P. Signorelli and R. Ghidoni, "Resveratrol as an anticancer nutrient: molecular basis, open questions and promises," The Journal of Nutritional Biochemistry, vol. 16, pp. 449-466, 2005. 
[9] M. Kitada and D. Koya, "Renal protective effects of resveratrol," Oxidative Medicine and Cellular Longevity, vol. 2013, Article ID 568093, 7 pages, 2013.

[10] R. Akgedik, Ş. Akgedik, H. Karamanlı et al., "Effect of resveratrol on treatment of bleomycin-induced pulmonary fibrosis in rats," Inflammation, vol. 35, p. 5, 2012.

[11] B. Catalgol, S. Batirel, Y. Taga, and N. K. Ozer, "Resveratrol: French paradox revisited," Frontiers in Pharmacology, vol. 3, p. 141, 2012.

[12] D. H. Kim, Y. J. Jung, J. E. Lee et al., "SIRT1 activation by resveratrol ameliorates cisplatin-induced renal injury through deacetylation of p53," American Journal of Physiology. Renal Physiology, vol. 301, pp. 427-435, 2011.

[13] A. Malhotra, S. Bath, and F. Elbarbry, "An organ system approach to explore the antioxidative, anti-inflammatory, and cytoprotective actions of resveratrol," Oxidative Medicine and Cellular Longevity, vol. 2015, Article ID 803971, 15 pages, 2015.

[14] S. Bradamante, L. Barenghi, and A. Villa, "Cardiovascular protective effects of resveratrol," Cardiovascular Drug Reviews, vol. 22, no. 3, pp. 169-188, 2004.

[15] S. Bandi, K. Sanka, and V. Bakshi, "Enhanced oral delivery of risperidone through a novel self-nanoemulsifying powder (SNEP) formulations: in-vitro and ex-vivo assessment," Journal of Microencapsulation, vol. 33, no. 6, pp. 544-553, 2016.

[16] S. Kapur, S. C. VanderSpek, and B. A. Brownlee, "Antipsychotic dosing in preclinical models is often unrepresentative of the clinical condition: a suggested solution based on in vivo occupancy," The Journal of Pharmacology and Experimental Therapeutics, vol. 305, no. 2, pp. 625-631, 2003.

[17] X. Zhang, Z. Zhang, W. Cheng, X. Mou, and G. P. Reynolds, "The effect of chronic antipsychotic treatment on sexual behaviour, hormones and organ size in the male rat," Journal of Psychopharmacology, vol. 21, no. 4, pp. 428-434, 2007.

[18] H. Zhao, X. Li, N. Li et al., "Long-term resveratrol treatment prevents ovariectomy-induced osteopenia in rats without hyperplastic effects on the uterus," British Journal of Nutrition, vol. 111, pp. 836-846, 2014.

[19] W. Junge, B. Wilke, A. Halabi, and G. Klein, "Determination of reference intervals for serum creatinine, creatinine excretion and creatinine clearance with an enzymatic and a modified Jaffé method," Clinica Chimica Acta, vol. 344, no. 1-2, pp. 137-148, 2004.

[20] O. Erel, "A novel automated method to measure total antioxidant response against potent free radical reactions," Clinical Biochemistry, vol. 37, no. 2, pp. 112-119, 2004.

[21] O. Erel, "A new automated colorimetric method for measuring total oxidant status," Clinical Biochemistry, vol. 38, no. 1, pp. 1103-1111, 2005.

[22] M. Harma, M. Harma, and O. Erel, "Oxidative stress in women with preeclampsia," American Journal of Obstetrics and Gynecology, vol. 192, pp. 656-657, 2005.

[23] U. Tas, M. Ayan, E. Sogut et al., "Protective effects of thymoquinone and melatonin on intestinal ischemia-reperfusion injury," Saudi Journal of Gastroenterology, vol. 21, pp. 284289, 2015.

[24] N. Can, O. Catak, B. Turgut et al., "Neuroprotective and antioxidant effects of ghrelin in an experimental glaucoma model," Drug Design, Development and Therapy, vol. 9, pp. 2819-2829, 2015.
[25] J. P. Domecq, G. Prutsky, A. Leppin et al., "Drugs commonly associated with weight change: a systematic review and metaanalysis," The Journal of Clinical Endocrinology and Metabolism, vol. 100, no. 2, pp. 363-370, 2015.

[26] D. B. Allison, J. L. Mentore, M. Heo et al., "Antipsychoticinduced weight gain: a comprehensive research synthesis," The American Journal of Psychiatry, vol. 156, no. 11, pp. 1686-1696, 1999.

[27] S. Gómez-Zorita, A. Fernández-Quintela, M. T. Macarulla et al., "Resveratrol attenuates steatosis in obese Zucker rats by decreasing fatty acid availability and reducing oxidative stress," British Journal of Nutrition, vol. 107, no. 2, pp. 202 210, 2012.

[28] A. Dal-Pan, S. Blanc, and F. Aujard, "Resveratrol suppresses body mass gain in a seasonal non-human primate model of obesity," BMC Physiology, vol. 10, no. 1, p. 11, 2010.

[29] J. A. Baur, K. J. Pearson, N. L. Price et al., "Resveratrol improves health and survival of mice on a high calorie diet," Nature, vol. 444, pp. 337-342, 2006.

[30] E. K. Naderali, "Obesity and cardiovascular dysfunction: a role for resveratrol," Obesity Research \& Clinical Practice, vol. 3, pp. 45-52, 2009.

[31] Y. J. Hwang, S. N. Dixon, J. P. Reiss et al., "Atypical antipsychotic drugs and the risk for acute kidney injury and other adverse outcomes in older adults: a population-based cohort study," Annals of Internal Medicine, vol. 161, no. 4, pp. $242-$ 248, 2014.

[32] K. L. Johansen, M. W. Smith, M. L. Unruh, A. M. Siroka, T. Z. O'Connor, and P. M. Palevsky, "Predictors of health utility among 60-day survivors of acute kidney injury in the veterans affairs/national institutes of health acute renal failure trial network study," Clinical Journal of the American Society of Nephrology, vol. 5, no. 8, pp. 1366-1372, 2010.

[33] C. W. Hsu, Y. Lee, C. Y. Lee, and P. Y. Lin, "Neurotoxicity and nephrotoxicity caused by combined use of lithium and risperidone: a case report and literature review," BMC Pharmacology and Toxicology, vol. 17, p. 59, 2016.

[34] Q. Hao, X. Xiao, J. Zhen et al., "Resveratrol attenuates acute kidney injury by inhibiting death receptor-mediated apoptotic pathways in a cisplatin-induced rat model," Molecular Medicine Reports, vol. 14, no. 4, pp. 3683-3689, 2016.

[35] D. F. Ding, N. You, X. M. Wu et al., "Resveratrol attenuates renal hypertrophy in early-stage diabetes by activating AMPK," American Journal of Nephrology, vol. 31, no. 4, pp. 363-374, 2010.

[36] J. H. Holthoff, Z. Wang, K. A. Seely, N. Gokden, and P. R. Mayeux, "Resveratrol improves renal microcirculation, protects the tubular epithelium, and prolongs survival in a mouse model of sepsis-induced acute kidney injury," Kidney International, vol. 81, pp. 370-378, 2012.

[37] L. Wu, Y. Zhang, X. Ma, N. Zhang, and G. Qin, "The effect of resveratrol on FoxO1 expression in kidneys of diabetic nephropathy rats," Molecular Biology Reports, vol. 39, no. 9, pp. 9085-9093, 2012.

[38] A. M. Osman, S. A. Telity, Z. A. Damanhouri et al., "Chemosensitizing and nephroprotective effect of resveratrol in cisplatin-treated animals," Cancer Cell International, vol. 15, p. 6, 2015.

[39] C. C. Chang, C. Y. Chang, Y. T. Wu, J. P. Huang, T. H. Yen, and L. M. Hung, "Resveratrol retards progression of diabetic nephropathy through modulations of oxidative stress, 
proinflammatory cytokines, and AMP-activated protein kinase," Journal of Biomedical Science, vol. 18, p. 47, 2011.

[40] P. Palsamy and S. Subramanian, "Resveratrol protects diabetic kidney by attenuating hyperglycemia-mediated oxidative stress and renal inflammatory cytokines via Nrf2-Keap1 signaling," Biochimica et Biophysica Acta, vol. 1812, no. 7, pp. 719-731, 2011.

[41] Ö. Demirpençe, B. Sevim, M. Yıldırım, N. A. Nurlu, D. Mert, and O. Evliyaoğlu, "Serum paraoxonase, TAS, TOS and ceruloplasmin in brucellosis," International Journal of Clinical and Experimental Medicine, vol. 7, no. 6, pp. 1592-1597, 2014.

[42] A. Aycicek and A. Ipek, "Maternal active or passive smoking causes oxidative stress in cord blood," European Journal of Pediatrics, vol. 167, p. 81, 2008.

[43] A. Dietrich-Muszalska and B. Kontek, "Lipid peroxidation in patients with schizophrenia," Psychiatry and Clinical Neurosciences, vol. 64, no. 5, pp. 469-475, 2010.

[44] Ö. Şehirli, A. Şakarcan, A. Velioğlu-Ögünç et al., "Resveratrol improves ifosfamide-induced Fanconi syndrome in rats," Toxicology and Applied Pharmacology, vol. 222, no. 1, pp. 33-41, 2007.

[45] Y. T. Wong, J. Gruber, A. M. Jenner, R. Ruan, and F. E. Tay, "Elevation of oxidative-damage biomarkers during aging in F2 hybrid mice: protection by chronic oral intake of resveratrol," Free Radical Biology \& Medicine, vol. 46, pp. 799-809, 2009.

[46] S. H. Hong, H. J. Lee, E. J. Sohn et al., "Anti-nephrolithic potential of resveratrol via inhibition of ROS,MCP-1, hyaluronan and osteopontin in vitro and in vivo," Pharmacological Reports, vol. 65, no. 4, pp. 970-979, 2013.

[47] E. Elmorsy, L. M. Elzalabany, H. M. Elsheikha, and P. A. Smith, "Adverse effects of antipsychotics on micro-vascular endothelial cells of the human blood-brain barrier," Brain Research, vol. 1583, pp. 255-268, 2014.

[48] I. E. da Cruz Jung, A. K. Machado, I. B. da Cruz et al., "Haloperidol and Risperidone at high concentrations activate an in vitro inflammatory response of RAW 264.7 macrophage cells by induction of apoptosis and modification of cytokine levels," Psychopharmacology, vol. 233, pp. 1715-1723, 2016.

[49] A. Ataie, M. Shadifar, and R. Ataee, "Polyphenolic antioxidants and neuronal regeneration," Basic and Clinical Neuroscience, vol. 7, no. 2, pp. 81-90, 2016.

[50] P. Kovacic and R. Somanathan, "Multifaceted approach to resveratrol bioactivity: focus on antioxidant action, cell signaling and safety," Oxidative Medicine and Cellular Longevity, vol. 3, no. 2, pp. 86-100, 2010.

[51] C. A. de la Lastra and I. Villegas, "Resveratrol as an antiinflammatory and anti-aging agent: mechanisms and clinical implications," Molecular Nutrition \& Food Research, vol. 49, no. 5, pp. 405-430, 2005.

[52] J. S. Modica-Napolitano, C. J. Lagace, W. A. Brennan, and J. R. Aprille, "Differential effects of typical and atypical neuroleptics on mitochondrial function in vitro," Archives of Pharmacal Research, vol. 26, no. 11, pp. 951-959, 2003.

[53] M. Gulec, H. Ozcan, E. Oral et al., "Nephrotoxic effects of chronically administered olanzapine and risperidone in male rats," Bulletin of Clinical Psychopharmacology, vol. 22, no. 2, pp. 139-147, 2012. 


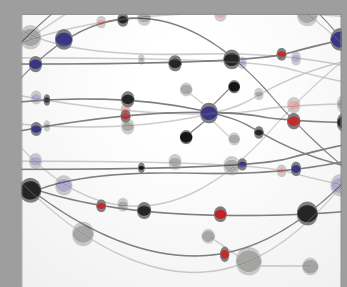

The Scientific World Journal
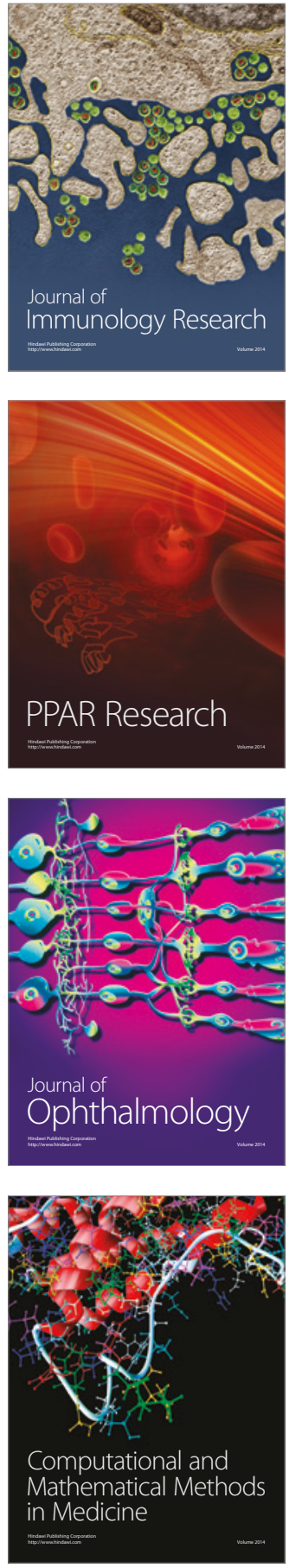

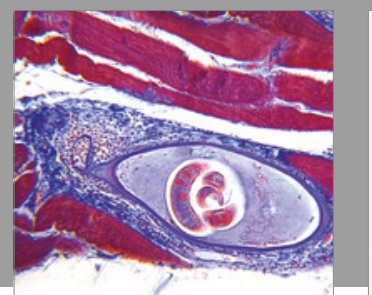

Gastroenterology Research and Practice
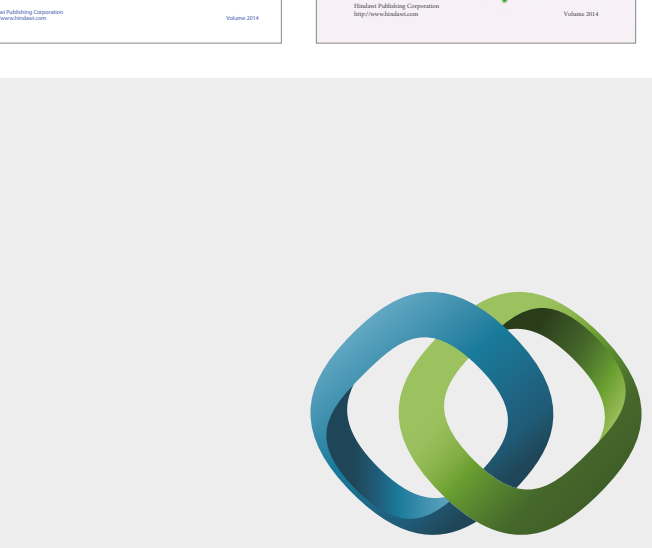

\section{Hindawi}

Submit your manuscripts at

https://www.hindawi.com
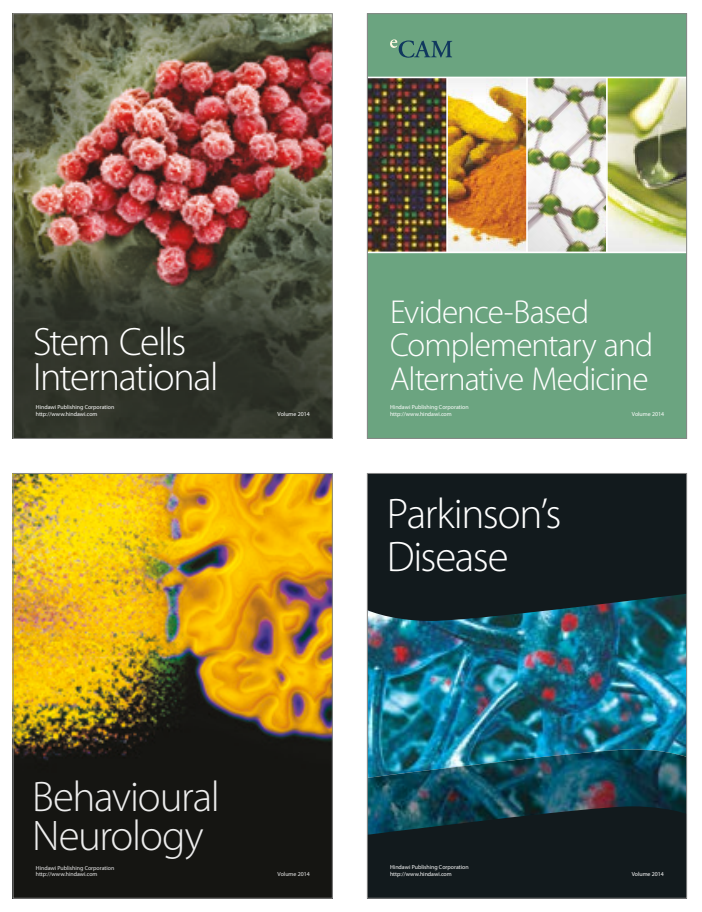
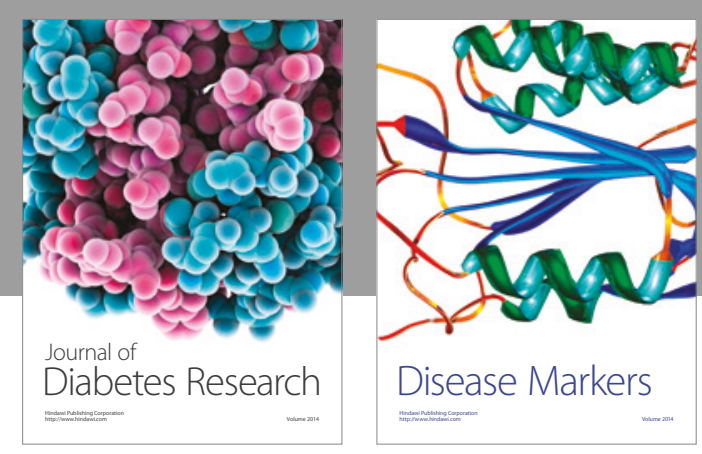

Disease Markers
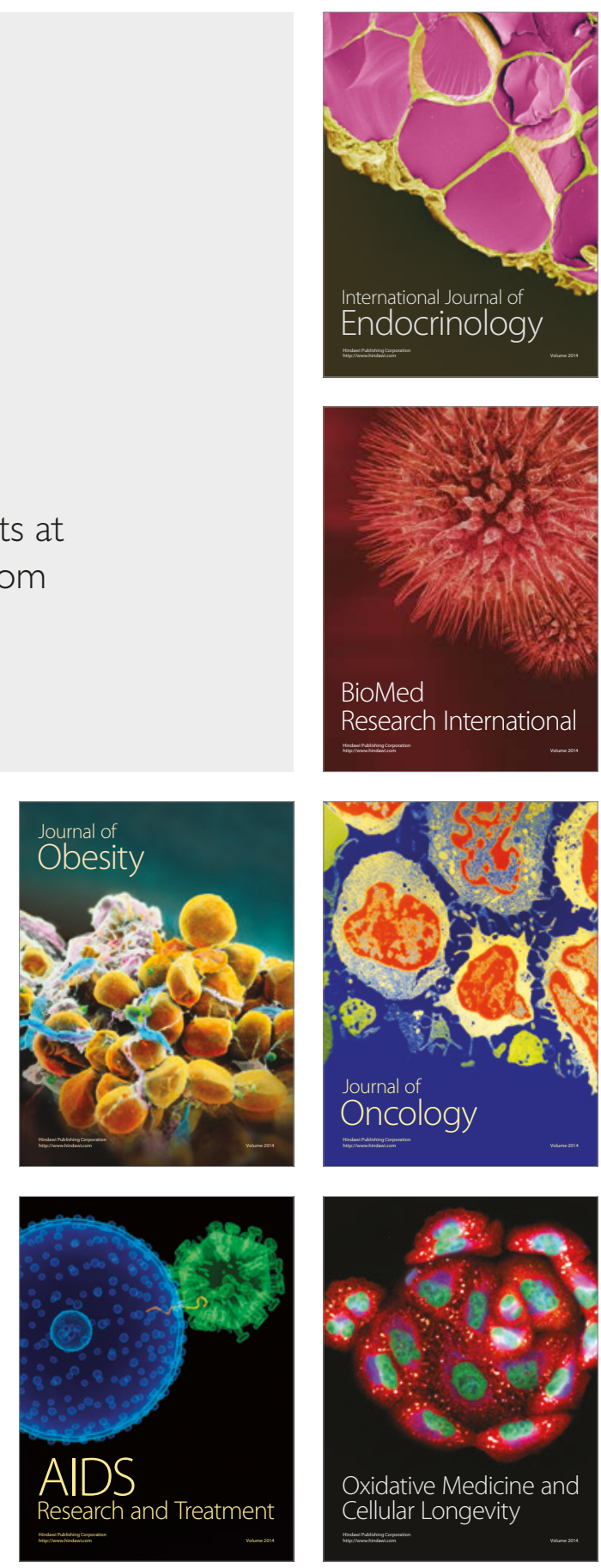